INFLAMMATION AND INFLAMMATORY BOWEL DISEASE

\title{
Distribution and partial characterisation of $\lg G$ Fc binding protein in various mucin producing cells and body fluids
}

\author{
K Kobayashi, H Ogata, M Morikawa, S lijima, N Harada, T Yoshida, W R Brown, \\ N Inove, Y Hamada, H Ishii, M Watanabe, T Hibi
}

See end of article for authors' affiliations

Correspondence to: Dr T Hibi, Department of Internal Medicine, School of Medicine, Keio University, 35

Shinanomachi, Shinjuku-ku, Tokyo 160-8582, Japan; thibi@sc.itc.keio.ac.jp

Accepted for publication 23 October 2001

\begin{abstract}
Background and aims: Mucus released from goblet cells is important in intestinal mucosal defence, and mucin glycoproteins are thought to be major components of mucus. Recently, we identified and cloned another component of human colonic mucus, IgG Fc binding protein (Fc $\gamma B P$ ). Fc $\gamma B P$ is immunologically distinct from known Fc $\gamma$ receptors and its structure contains repeated cysteine rich unit sequences resembling those present in mucins. In this work, we assessed the tissue distribution of Fc $\gamma \mathrm{BP}$, its binding activity in various body fluids, and its ability to inhibit complement mediated haemolysis. Methods: Immunohistochemical localisation of $\mathrm{Fc} \gamma \mathrm{BP}$, using monoclonal antibodies against Fc $\gamma \mathrm{BP}$ (K9 or K17) and labelled lgG, was conducted in various mucin producing tissues: colon, small intestine, stomach, gall bladder, cystic duct, choledochus, bronchus, submandibular gland, conjunctiva, and cervix uteri. The binding activity of $\mathrm{Fc} \gamma \mathrm{BP}$ in mucus extracted from colon, gastric juice, bile, nasal discharges, saliva, sputum, and tears was also examined by immunodotblot and immunoprecipitation using these monoclonal antibodies. Inhibition of complement mediated haemolysis by Fc $\gamma B P$ was investigated using sheep red blood cells (SRBC) and anti-SRBC lgG.

Results: The immunohistochemical study revealed that mucin secreting cells in the colon, small intestine, gall bladder, cystic duct, choledochus, bronchus, submandibular gland, and cervix uteri contained FcyBP, and immunodotblot and immunoprecipitation analysis using $\lg G$ and monoclonal antibodies demonstrated that the fluids secreted by these cells were capable of binding lgG. Mucin producing cells of the conjunctiva did not express FcyBP molecules or bind to lgG. The surface mucus cells in the stomach were variably positive for FcyBP. Perhaps because of proteolytic degradation, $\mathrm{Fc \gamma BP}$ in gut lavage fluid did not have IgG binding activity, although this activity was present in the mucus covering the colon. FcyBP suppressed complement mediated haemolysis of SRBC.

Conclusions: FcyBP is widely expressed on mucosal surfaces and in external secretions. It is functionally intact in several fluids. These findings lend support to the concept that Fc $\gamma B P$ is an important component of mucosal immunological defences.
\end{abstract}

$\uparrow$ ag he epithelium of the gastrointestinal mucosa is a barrier against potentially injurious luminal agents, including acid, enzymes, toxins, and infectious organisms. Disruption of this barrier is a feature of common and important gastrointestinal disorders, including inflammatory bowel diseases and peptic ulcer. ${ }^{1-3}$ Several general and specific protective factors are thought to contribute to maintenance of the barrier. Goblet cells are abundant constituents of the surface epithelium within the small and large intestine that are thought to play an important role in mucosal protection. ${ }^{3-6}$ Mucus released from goblet cells is important for intestinal mucosal defence but the mechanisms of this action have been poorly understood. However, recent advance in molecular biology and biochemistry have provided new insights into the functional role of mucins in the gastrointestinal tract. Based on knowledge of their structure and expression patterns, it is likely that these molecules play an important role in barrier function. In addition to presenting large extended heavily glycosylated mucin domains on the cell surface, highly conserved structural features suggest that an ability to shed the extracellular mucin domain and to report via cytoplasmic domain signalling are important facets of these cell surface molecules. The signalling pathways triggered by cell surface mucins appear to show that MUCl can activate the ras pathway via $\mathrm{Grb} 2^{7}$ and interact with $\beta$-catenin, an important modulator of cell adhesion and growth. ${ }^{89}$ Interactions between secreted mucins and bacteria have been well characterised, ${ }^{10}$ and it is possible that interaction with bacteria is an important part of cell surface mucin function. A recent study utilising $\mathrm{CHO}$ cells stably expressing Mucl mucin ${ }^{11}$ demonstrated phosphorylation of the Mucl cytoplasmic domain following adhesion of Pseudomonas aeruginosa to the extracellular domain of this mucin. Furthermore, MUC3, MUC4, and MUC12 all contain two cysteine rich epidermal growth factor-like motifs, and the function of epidermal growth factor-like motifs in these mucins is unclear although there is evidence implicating these domains in epithelial growth modulation. ${ }^{12}$ At present, 13 genes have been identified. ${ }^{3}{ }^{13}$ Recently, we described a unique intestinal binding site for the Fc region of IgG associated with goblet cells in the human intestine ${ }^{14}$ and produced several monoclonal antibodies against the binding site. ${ }^{14}$ This binding site is immunologically distinct from known Fc $\gamma$ receptors on leucocytes, and appears to be secreted with mucus into the intestinal lumen. ${ }^{14-16}$ Immunoblot analysis, using our monoclonal antibodies, revealed a molecular weight for IgG Fc binding protein $(\mathrm{Fc} \gamma \mathrm{BP})$ of $>200 \mathrm{kDa}$ under non-reduced conditions

Abbreviations: FcyBP, lgG Fc binding protein; HRP, horseradish peroxidase; $\mathrm{mAb}$, monoclonal antibody; $\mathrm{PBS}$, phosphate buffered saline; DAB, 3-3'-diaminobenzidine; EDTA, ethylenediaminetetraacetic acid; PMSF, phenylmethylsulphonyl fluoride; SRBC, sheep red blood cells. 

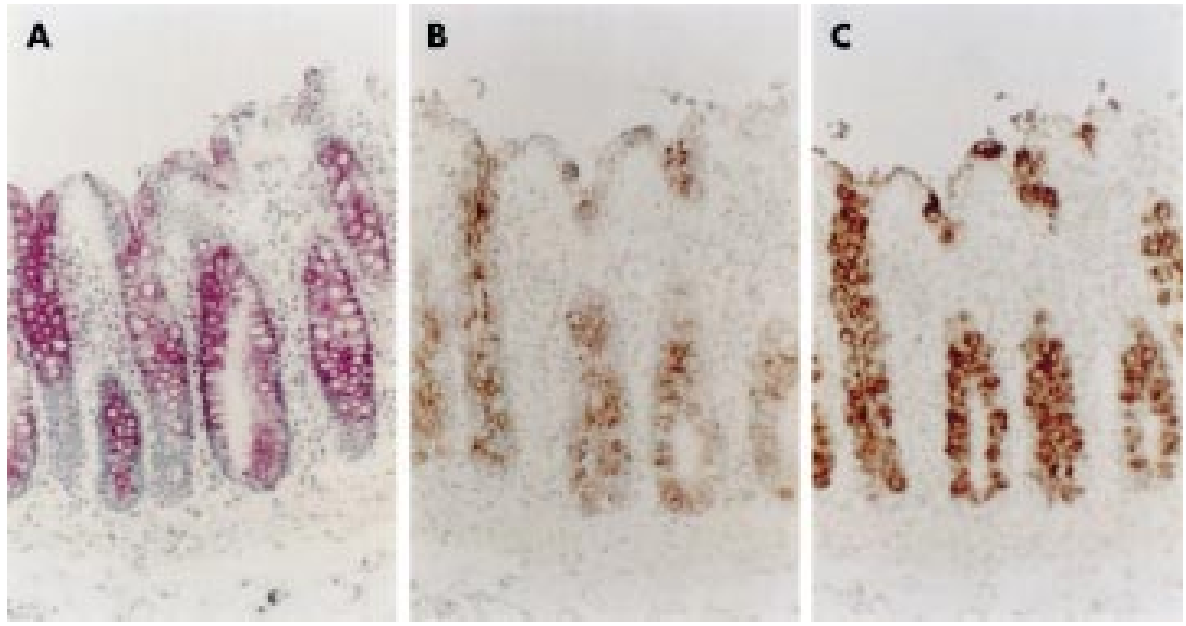

Figure 1 Micrographs of serial cryostat sections of human colonic mucosa. Normal colonic mucosa was stained by periodic acid-Schiff (PAS) $(A)$, reacted with horseradish peroxidase (HRP)-lgG (B), or reacted with the monoclonal antibody $(\mathrm{mAb}) \mathrm{K} 9$ (C) (100x). The sections were counterstained with methylgreen. PAS, HRP-lgG, and mAb K9 stained goblet cells in the sections.

(reactivity with K9) and of 70-80 kDa under reduced conditions (reactivity with K17) for Fc $\gamma B P^{9}$ Cloning of a cDNA for human Fc $\gamma \mathrm{BP}$ revealed mRNA with a coding region of 17 and $16.2 \mathrm{~kb}$, respectively. The amino acid sequence showed homology to protein components of mucins, such as MUC2 and prepro-von Willebrand factor. ${ }^{17}$

As mucin glycoproteins, which are the major components of mucus, are secreted from various body tissues other than the intestine, ${ }^{18}{ }^{19}$ we hypothesised that Fc $\gamma \mathrm{BP}$ is also present in other mucin secreting tissues and thus is extensively involved in mucosal protection.

In the present work, we used our monoclonal antibodies against Fc $\gamma B P$ and labelled IgG to study the distribution of Fc $\gamma B P$ in various mucin producing cells and body fluids and its binding activity with IgG.

\section{MATERIALS AND METHODS}

\section{Tissue samples and products}

Five specimens from normal human colon, three from the small intestine, and five from the stomach were obtained at surgical resection. Four specimens of gall bladder, cystic duct, choledochus, and bronchus were obtained at autopsy. Five specimens of the nasal mucosa, three of the submandibular gland, five of the conjunctiva, and three of the cervix uteri were obtained at surgical resection. Written informed consent was obtained from all patients, and all experiments were approved by the Keio University Hospital Committee on Human Subjects.

Horseradish peroxidase (HRP) type 6, 3-3'-diaminobenzidine (DAB), ethylenediamine tetraacetic acid (EDTA), phenylmethylsulphonyl fluoride (PMSF), soybean trypsin inhibitors, Sepharose 4B, magnesium chloride, and calcium chloride were purchased from Sigma Chemical Co (St Louis, Missouri, USA). Tissue-Tek OCT compound was purchased from Miles Laboratories, Inc., (Naperville, Illinois, USA). Normal mouse IgG and HRP-goat $\mathrm{F}\left(\mathrm{ab}^{\prime}\right) 2$ anti-mouse IgG were purchased from Zymed Laboratories, Inc. (San Francisco, California, USA). Nitrocellulose paper and polysorbate (Tween 20) were purchased from Bio-Rad Laboratories (Richmond, California, USA). Immunobeads (goat IgG antimouse IgG), sheep red blood cells (SRBC), anti-SRBC rabbit IgG, and nonimmunised rabbit IgG were purchased from Cappel (West Chester, Pennsylvania, USA). Rabbit complement was purchased from Cedarlane (Hornby, Ontario, Canada).

\section{Immunohistochemical and histochemical methods}

Human IgG was purified from the sera of three healthy subjects using 50\% ammonium sulphate precipitation and DEAE cellulose ion exchange chromatography. HRP conjugated IgG was prepared with conjugation to HRP type 6, as previously described. ${ }^{14}$ All tissue specimens were embedded in Tissue-Tek OCT compound and snap frozen. Fresh frozen tissues were sectioned into $6 \mu \mathrm{m}$ thick samples on a cryostat microtome. After fixation in cold $100 \%$ ethanol for five minutes, sections were washed with phosphate buffered saline (PBS). To identify mucus cells with IgG binding activity, sections were incubated with HRP-IgG $(20 \mu \mathrm{g} / \mathrm{ml})$ for one hour at room temperature using our previously described methods. ${ }^{14}$ Using another method of detecting FcyBP, sections were incubated overnight at $4^{\circ} \mathrm{C}$ with the supernatant of mouse IgG monoclonal antibody $(\mathrm{mAb})$ against Fc $\gamma \mathrm{BP}(\mathrm{K} 9)$ and washed with PBS. This antibody reacts with human Fc $\gamma \mathrm{BP}$ and was found to be the most suitable for immunohistochemical staining among 14 mAbs which we established in a previous study. Then, sections were reacted with HRP-goat $\mathrm{F}\left(\mathrm{ab}^{\prime}\right) 2$ anti-mouse IgG for one hour at room temperature. After further washing, peroxidase activity was developed by reaction with $\mathrm{DAB}$ solution and counterstained with methyl green. ${ }^{16} 20$

\section{Sample preparation for immunodotblot and immunoprecipitation}

An enriched fraction of FcyBP was prepared from a homogenate of human colonic epithelial cells, as described previously. ${ }^{14}$ Briefly, epithelial cells were dissociated in EDTA containing buffer and disrupted by a polytron (Kinematic type PT200D; Kinematica GMBD, Lucerne, Switzerland) on ice. After sonication, the homogenate was centrifuged at $10000 \mathrm{~g}$ for 15 minutes. The resulting supernatant (designated $10 \mathrm{~K}$ homogenate) was used as a positive control in experiments to detect the presence of Fc $\gamma \mathrm{BP}$ in body fluids. Mucus covering the colon was scraped very gently from the colonic mucosa that was obtained at operation, diluted with PBS containing $10 \mathrm{mM}$ EDTA, 2 mM PMSF, and 0.005\% soybean trypsin inhibitors, stirred, and centrifuged at $700 \mathrm{~g}$ for 10 minutes. The supernatant was centrifuged at $10000 \mathrm{~g}$ for 15 minutes. This supernatant was used for immunoblot and immunoprecipitation experiments. Nasal discharges and sputum were prepared in the same manner as mucus covering the colon. Gut lavage fluid and gastric juice were collected at endoscopy. Bile was collected through a drainage tube from patients with common bile duct cancer. The gut lavage fluid, gastric juice, bile, tears, and saliva were centrifuged at $10000 \mathrm{~g}$ for 15 minutes before use.

\section{Immunodotblot for detection of Fc $\gamma B$ P}

These supernatants $(2 \mu \mathrm{l})$ were dropped onto nitrocellulose paper and dried. Nitrocellulose paper was washed three times 

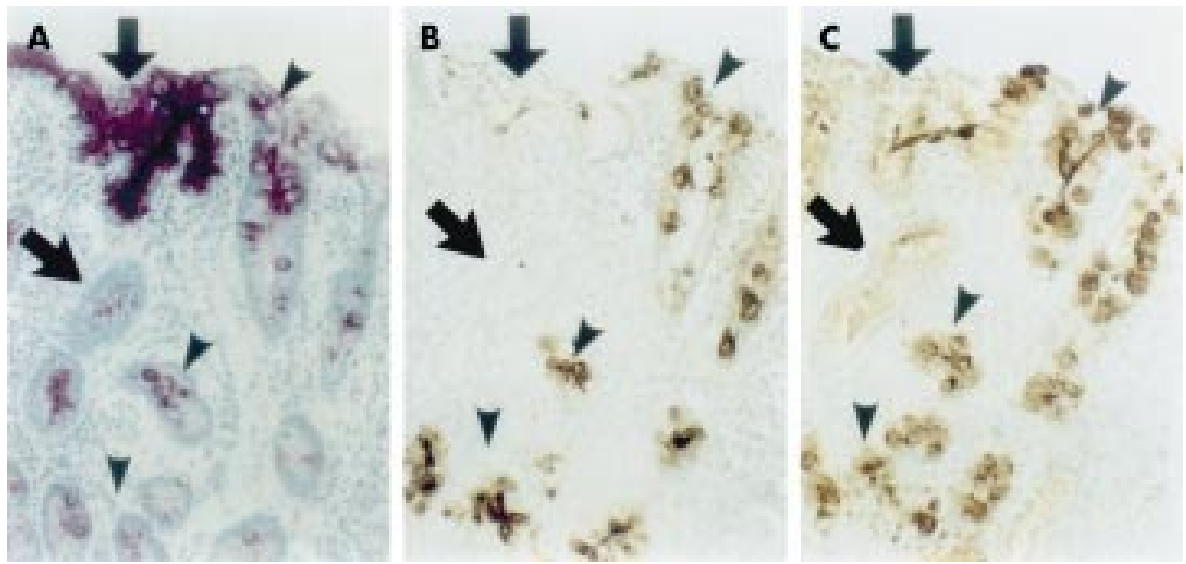

Figure 2 Micrographs of serial cryostat sections of human stomach. Normal surface mucus cells (arrows) face the portion of intestinal metaplasia. (A) Normal surface mucus cells (arrows) and mucin producing cells in intestinal metaplasia (arrowheads) were stained by periodic acid-Schiff (arrowheads show the same portion on serial sections). (B) Normal surface mucus cells (arrows) were not stained by horseradish peroxidase-lgG whereas mucin producing cells of goblet cell-like intestinal metaplasia were stained. (C) Normal surface mucus cells (arrows) were weakly stained by the monoclonal antibody (mAb) K9. Mucin producing cells of intestinal metaplasia (arrowheads) were clearly stained by mAb K9 (200x).

with Tris buffer solution (20 mM Tris $\mathrm{HCl} \mathrm{pH} \mathrm{7.4/0.15} \mathrm{M} \mathrm{NaCl)}$ containing $0.5 \%$ Tween 20 (TBS-T). Nitrocellulose paper was blocked with TBS-T containing $1 \%$ skimmed milk powder for 30 minutes to decrease non-specific binding and washed three times with TBS-T. Next, the paper was reacted with the mAbs K9, K17, a control IgG1 mAb (OE5) which was prepared in our laboratory against human asialoglycoprotein receptor, normal mouse IgG, or TBS-T for one hour at $37^{\circ} \mathrm{C}$. After washing with TBS-T three times, the nitrocellulose paper was incubated with HRP-goat $\mathrm{F}\left(\mathrm{ab}^{\prime}\right) 2$ anti-mouse IgG for one hour at $37^{\circ} \mathrm{C}$. Nitrocellulose paper was washed with TBS-T three times and developed with DAB solution. More than two samples of each fluid were examined.

\section{Immunoprecipitation for detection of $\mathrm{Fc} \gamma \mathrm{BP}$}

The supernatants prepared for immunodotblot were diluted twice with TBS-T containing $10 \mathrm{mM}$ EDTA, $2 \mathrm{mM}$ PMSF, and $0.005 \%$ soybean trypsin inhibitors. Each diluted supernatant
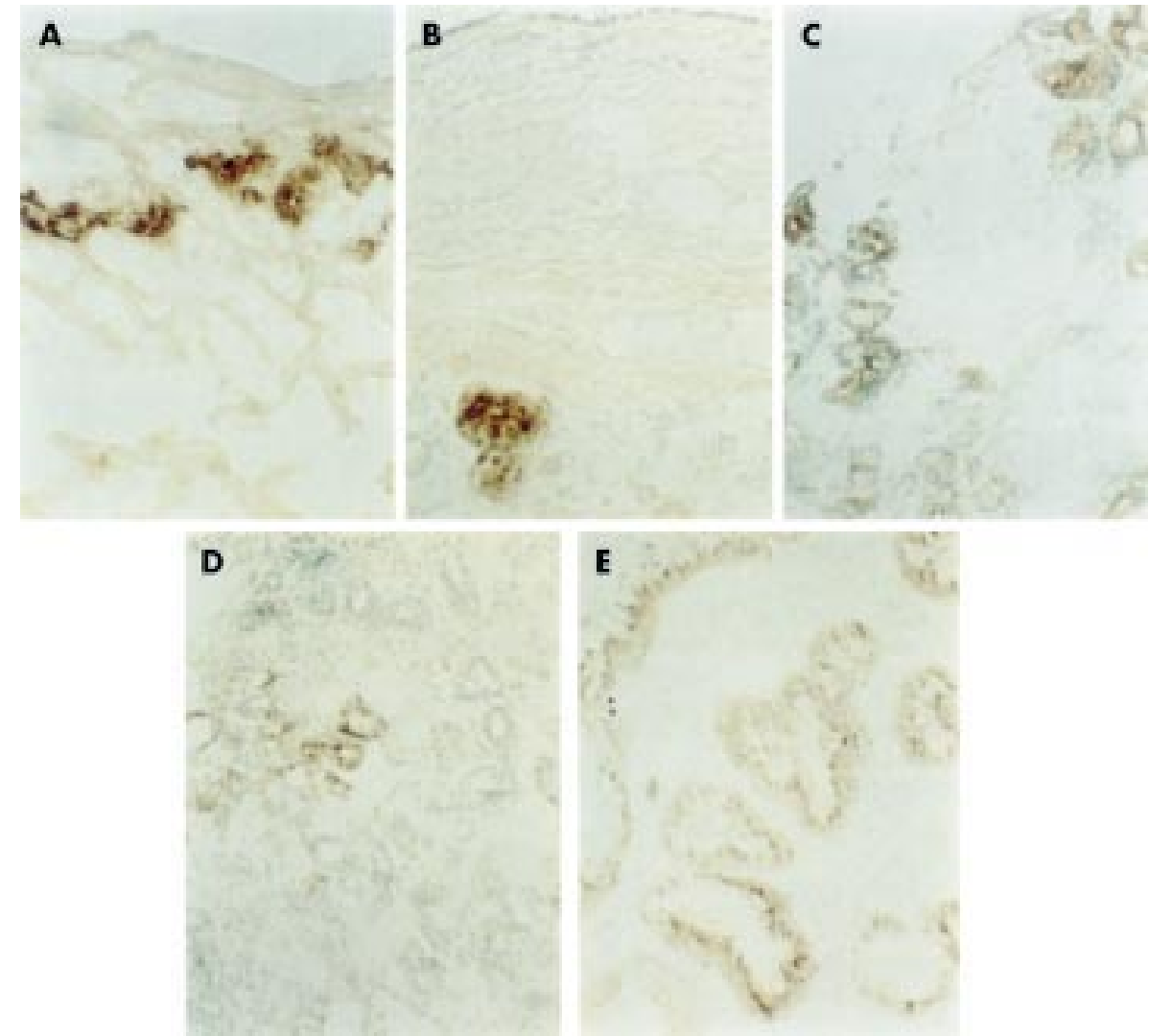

Figure 3 Micrographs of mucin producing cells of various organs, immunohistochemically stained by horseradish peroxidase (HRP)-lgG and monoclonal antibodies (mAbs). Mucin producing cells of mucus glands in the choledochus (A) and bronchial glands (B) were stained with HRP-lgG. Mucin producing cells in the nasal glands (C), acinus of the submandibular gland (D), and cervical glands of the uterus (E) were stained by the mAb K9 (200x). Mucin producing cells stained by periodic acid-Schiff were all positive for HRP-lgG and mAbs. 


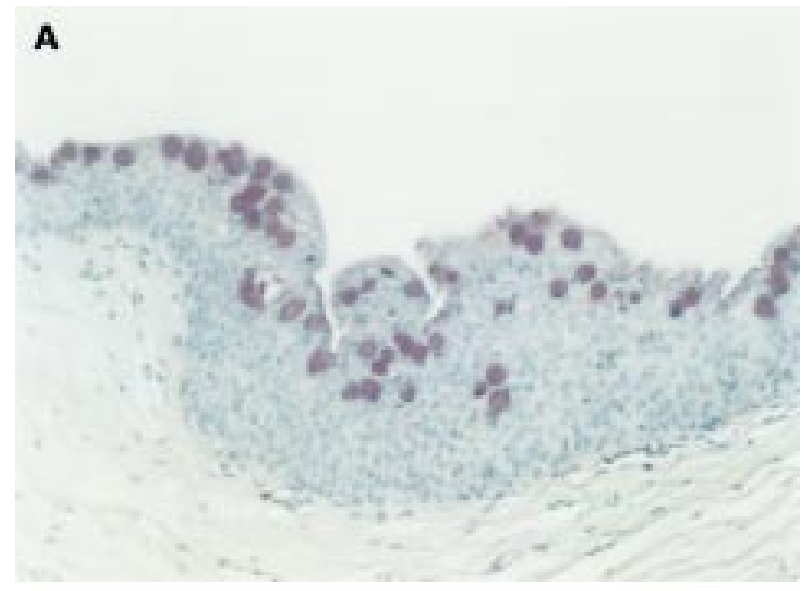

B

Figure 4 Micrographs of cryostat sections of human conjunctiva stained by periodic acid-Schiff (PAS) (A) and immunohistochemically reacted with the monoclonal antibody ( $\mathrm{mAb}$ ) K9 (B). PAS positive mucin producing cells were not stained by the mAb K9 (200x).

( $1 \mathrm{ml}$ ) was incubated with $100 \mu \mathrm{l}$ of Sepharose 4B for 30 minutes at room temperature to remove non-specific binding. After centrifugation at $2000 \mathrm{~g}$ for one minute, $500 \mu \mathrm{l}$ of each supernatant were applied directly to $50 \mu \mathrm{l}$ of immunobeads and incubated for one hour at room temperature for the detection of IgG binding Fc $\gamma \mathrm{BP}$ (subsequently referred to as active Fc $\gamma \mathrm{BP}$ ). We had previously shown that human Fc $\gamma \mathrm{BP}$ binds goat IgG. ${ }^{14} \mathrm{In}$ other experiments, $2 \mu \mathrm{l}$ of $\mathrm{mAb} \mathrm{K} 9(2 \mathrm{mg} / \mathrm{ml}), \mathrm{Kl} 7$ ( $3 \mathrm{mg} / \mathrm{ml})$, or normal mouse IgG $(3 \mathrm{mg} / \mathrm{ml})$ were added to another $500 \mu \mathrm{l}$ of each supernatant and then incubated for one hour. Immunobeads $(50 \mu \mathrm{l})$ were added to each of the mixtures and incubated for one hour at room temperature. After all immunobeads were washed five times, $70 \mu \mathrm{l}$ of sodium dodecyl sulphate sample buffer with mercaptoethanol were added to each immunobead preparation and boiled for two minutes, and the dissociated protein was resolved in $8 \%$ sodium dodecyl sulphatepolyacrylamide gel electrophoresis. The positions of molecular weight standards were identified by rainbow marker (Bio-Rad Kaleidoscope Protein Standards) in each experiment. The protein was transferred to nitrocellulose papers and incubated with mAb K17 after blocking with TBS-T containing 1\% skimmed milk powder. These papers were reacted with HRP-goat $F\left(a b^{\prime}\right) 2$ antimouse IgG and developed with DAB solution. Nitrocellulose papers were washed three times with TBS-T between each incubation step.

\section{Inhibition of complement mediated haemolysis by $\mathbf{F} \mathbf{c} \gamma \mathbf{B P}$}

SRBC were washed three times with physiological saline containing $0.1 \%$ gelatine, $3 \mathrm{mM}$ barbiturate, $1.8 \mathrm{mM}$ barbital, 0.5
Table 1 Detection of $\lg G$ Fc binding protein by horseradish peroxidase (HRP)-lgG and monoclonal antibodies (mAbs) K9 and K17

\begin{tabular}{|c|c|c|c|}
\hline & HRP-IgG & K9 & $\mathrm{K} 17$ \\
\hline Colon $(5 / 5)$ & + & + & + \\
\hline Small intestine $(3 / 3)$ & + & + & + \\
\hline \multicolumn{4}{|l|}{ Stomach } \\
\hline Surface mucus cells* & - & $\sim+$ & $\sim+$ \\
\hline Intestinal metaplasia $(5 / 5)$ & + & + & + \\
\hline Gall bladder (4/4) & + & + & + \\
\hline Cystic duct (4/4) & + & + & + \\
\hline Choledochus (4/4) & + & + & + \\
\hline Bronchus (mucus gland) $(4 / 4)$ & + & + & + \\
\hline Nasal mucosa (mucus gland) (4/4) & + & + & + \\
\hline Submandibular gland (4/4) & + & + & + \\
\hline Conjunctiva $(5 / 5)$ & - & - & - \\
\hline Cervix uteri $(3 / 3)$ & + & + & + \\
\hline
\end{tabular}

* Surface mucus cells of the stomach showed negative stain with HRP-lgG, but in three of five samples these cells had a weak stain with the $\mathrm{mAb}$ and in two of five there was no stain.

$\mathrm{mM}$ magnesium chloride, and $0.15 \mathrm{mM}$ calcium chloride. Anti-SRBC rabbit IgG ( $100 \mu \mathrm{l} ; 300 \mu \mathrm{g} / \mathrm{ml})$ was added to $1 \mathrm{ml}$ of SRBC $(1 \times 108 / \mathrm{ml})$ and incubated for one hour at $37^{\circ} \mathrm{C}$. Next, $100 \mu \mathrm{l}$ of the rich fraction of Fc $\gamma B P$ (10K homogenate) (150 $\mu \mathrm{g} / \mathrm{ml}$ ) or PBS alone containing $10 \mathrm{mM}$ EDTA, $2 \mathrm{mM}$ PMSF, and $0.005 \%$ soybean trypsin inhibitors were added to this solution and incubated for one hour at $37^{\circ} \mathrm{C}$. Then, $200 \mu \mathrm{l}$ of rabbit complement $(4 \mathrm{mg} / \mathrm{ml})$, or PBS as a negative control, were reacted with this solution for one hour at $37^{\circ} \mathrm{C}$. The reaction was stopped by chilling on ice. The solution was centrifuged at $1500 \mathrm{~g}$ for five minutes. The optical density of haemoglobin in supernatants was read at $541 \mathrm{~nm}$. To further test the specificity of Fc $\gamma \mathrm{BP}$ in inhibiting haemolysis, two other control experiments were conducted. Firstly, an excess of non-immune rabbit IgG $(600 \mu \mathrm{g} / 100 \mu \mathrm{l})$ was incubated with the $10 \mathrm{~K}$ homogenate for one hour at $37^{\circ} \mathrm{C}$. This solution was then added to SRBC bound anti-SRBC rabbit IgG and the complement was reacted. Secondly, to inactivate the function of FcyBP, the 10K homogenate was boiled for five minutes before being added to the haemolysis reaction mixture.

\section{RESULTS}

Localisation of FcyBP in tissues by immunohistochemistry and reaction with labelled IgG

As in our previous work, ${ }^{1420}$ goblet cells in the normal colon and small intestine were stained by HRP-IgG and mAbs K9 and K17, but not by the irrelevant mAb OE5 (fig 1). In experiments to identify FcyBP positive cells in other tissues, mucin producing cells were detected by periodic acid-Schiff staining, and normal colon was used as a positive control for HRP-IgG binding and mAb staining. Surface mucus cells in the apparently normal stomach adjacent to intestinal metaplasia did not react with HRP-IgG, but these cells were weakly stained with the mAb in three of five samples. In contrast, mucin producing cells of goblet cell-like intestinal metaplasia of the stomach were positive for HRP-IgG binding as well as K9 and K17 staining (fig 2). Concerning the classification of intestinal metaplasia, two specimens showed the complete form of intestinal metaplasia and the three others the incomplete form. We found that both complete and incomplete forms of intestinal metaplasia were clearly positive for Fc $\gamma \mathrm{BP}$ and there was no difference in staining pattern between the two. We also examined three endoscopically biopsied samples of apparently normal stomach. All were negative for HRP-IgG binding and showed little or no staining (data not shown). All mucin producing cells of mucus glands in the choledochus, cystic duct, gall bladder near the cystic duct, and bronchial glands were 


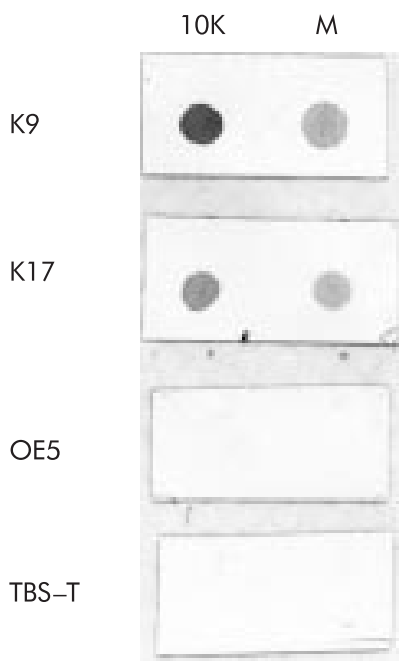

Figure 5 Immunodotblot of the 10K homogenate or mucus covering the colon. The 10K homogenate or mucus covering the colon were dropped onto nitrocellulose paper and the paper was incubated with the monoclonal antibodies (mAbs) K9, K17, OE5 (irrelevant $\mathrm{mAb}$ ), or TBS-T. Blots were then reacted with horseradish peroxidase-goat $F\left(a b^{\prime}\right) 2$ antimouse $\lg G$. K9 and $K 17$ reacted positively but OE5 and TBS-T did not. 10K, 10K homogenate; $M$, mucus covering the colon.

stained with HRP-IgG as well as with mAbs K9 and K17. In addition, all mucin producing cells in the nasal glands, acinus of the submandibular glands, and cervical glands of the uterus were also positive for HRP-IgG binding and mAb staining (fig 3) while mucin producing cells of conjunctivae were negative for reaction with these reagents (fig 4, table 1 ).

\section{Immunodotblot for detection of $\mathrm{Fc} \gamma \mathrm{BP}$ in secretions}

Dots of the $10 \mathrm{~K}$ homogenate and mucus covering the colon were stained by K9 and K17 but not by control OE5 or TBS-T (fig 5). In addition, normal mouse IgG showed no staining. Bile, nasal discharges, saliva, and sputum were positive for reaction with mAbs K9 and K17 but tears were negative (fig 6). Among five samples of gastric juice examined, two were positive but three were negative for reaction with both mAbs. The $\mathrm{pH}$ of the juices with positive staining was more than 6, whereas the $\mathrm{pH}$ of non-reactive juice was less than 3 .

\section{Immunoprecipitation and immunoblot for characterisation of $\mathrm{Fc} \gamma \mathrm{BP}$}

In each experiment, the 10K homogenate was used as a positive control. Active Fc $\gamma \mathrm{BP}$ capable of binding IgG was detected by direct incubation with immunobeads (goat $\operatorname{IgG}$ ). We have already demonstrated that human FcyBP can also bind to goat IgG. ${ }^{14}$ Immunoprecipitated active $\mathrm{Fc} \gamma \mathrm{BP}$ was visualised at 70-80 kDa by immunoblot analysis with the mAb K17 under

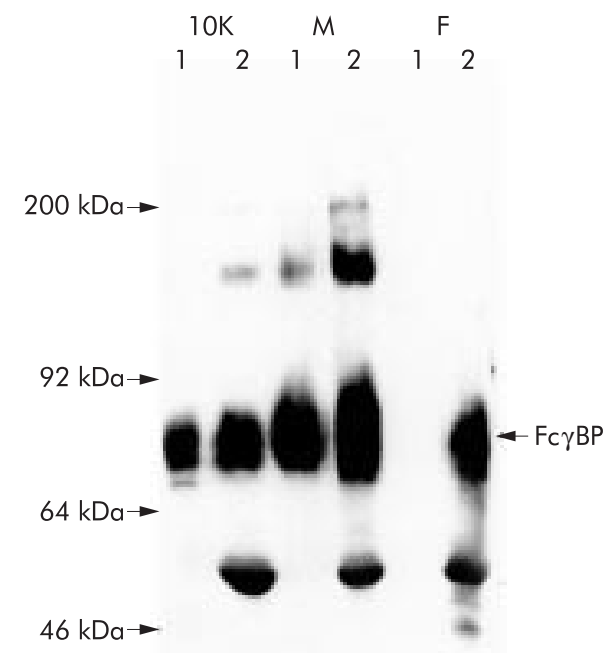

Figure 7 Immunoprecipitation and immunoblot of the 10K homogenate (10K), mucus covering the colon (M), and gut lavage fluid $(F)$. Lane 1: Immunoblot from direct immunoprecipitation with immunobeads (goat lgG antimouse lg $\mathrm{G}$ ). The $70-80 \mathrm{kDa}$ band is present on the nitrocellulose paper of the 10K homogenate and mucus covering the mucosa under reduced conditions but not on that of gut lavage fluid. Active lgG Fc binding protein (FcyBP) was captured by goat lgG via the Fc portion. Lane 2: Immunoblot from immunoprecipitation with a mixture of the monoclonal antibodies

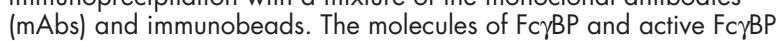
were visualised on the paper. The $10 \mathrm{~K}$ homogenate and mucus covering the colon had a 70-80 kDa band similar to that in lane 1. Gut lavage fluid had the 70-80 kDa band and a few smaller bands. The bands between 92 and $200 \mathrm{kDa}$ are probably doublets and triplets of the $70-80 \mathrm{kDa}$ band. Bands at about $50 \mathrm{kDa}$ are heavy chains of the mAbs. From this result, it is evident that the 10K homogenate and mucus covering the colon contain active Fc $\gamma B P$ but gut lavage fluid contains only fragments of FcyBP. Arrows show markers of 200 kDa (myosin), $92 \mathrm{kDa}$ (phosphorylase), $64 \mathrm{kDa}$ (bovine serum albumin), and $46 \mathrm{kDa}$ (ovalbumin).

reduced conditions. The mAbs $\mathrm{K} 9$ and $\mathrm{K} 17$ were able to capture the molecules of FcyBP through the Fab region of the $\mathrm{mAb}$ besides the Fc region of the mAbs and goat IgG. In case of the $10 \mathrm{~K}$ homogenate of the colon, immunoprecipitation with the mAbs identified the same $70-80 \mathrm{kDa}$ band on the immunoblot as the active FcyBP with the immunobeads. Mucus covering the colon yielded the same results as those for the $10 \mathrm{~K}$ homogenate. But no specific bands were found using normal mouse IgG. Gut lavage fluid did not show the 70-80 kDa band when it was immunoprecipitated only with immunobeads. However, the mAbs identified the 70-80 kDa band of FcyBP recognised by mAb K17 on the immunoblot (fig 7). Nasal discharges, sputum, and bile had almost the same IgG

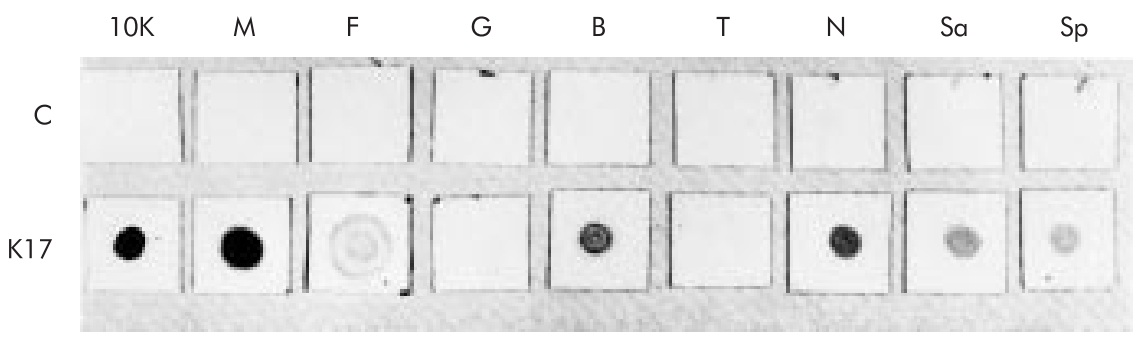

Figure 6 Immunodotblot of various body fluids. Body fluids were dropped onto nitrocellulose paper and the paper was incubated with the monoclonal antibody (mAb) K17 and reacted with horseradish peroxidase-goat F(ab')2 antimouse lgG. Molecules of the lgG Fc binding protein (FcyBP) recognised by the mAb K17 were present in fluids, except gastric juice and tears. Incubation with TBS-T instead of mAb K17 showed no stain (C). 10K, 10K homogenate; $M$, mucus covering the colon; $F$, gut lavage fluid; $G$, gastric juice; $B$, bile; $T$, tears; $N$, nasal discharges; Sa, saliva; Sp, sputum. 


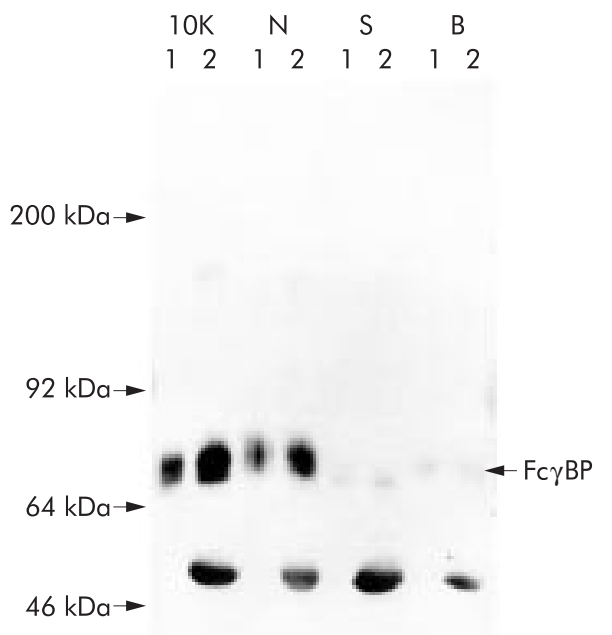

Figure 8 Immunoprecipitation and immunoblot of nasal discharges $(\mathrm{N})$, sputum (S), and bile (B). Lane 1: Immunoblot from direct immunoprecipitation with immunobeads (goat lgG anti-mouse lgG). Lane 2: Immunoblot from immunoprecipitation with a mixture of the monoclonal antibodies (mAbs) and immunobeads. Nasal discharges had the 70-80 kDa band similar to that of the 10K homogenate in both lanes 1 and 2. Sputum and bile showed the same faint band as the $10 \mathrm{~K}$ homogenate. Thus these fluids contain active FcyBP.

binding activity and the same molecular size of $\mathrm{Fc} \gamma \mathrm{BP}$ as those of the 10K homogenate (fig 8).

\section{Inhibition of complement mediated haemolysis by Fc $\gamma B P$ (fig 9)}

SRBC reacted with anti-SRBC rabbit IgG were haemolysed after incubation with complement. Addition of the $10 \mathrm{~K}$ homogenate containing Fc $\gamma$ BP significantly $(p<0.01)$ inhibited complement mediated haemolysis. Addition of excess non-immune rabbit IgG (to bind the FcyBP) to the $10 \mathrm{~K}$ homogenate before incubation with complement abolished the inhibitory effect of Fc $\gamma B$ P. Addition of heat treated (inactivated) FcyBP to the reaction had no inhibitory effect on haemolysis. From these results, we conclude that the rich fraction of Fc $\gamma B$ PP specifically inhibited complement mediated haemolysis of SRBC.

\section{DISCUSSION}

Intestinal mucus is probably critical in protecting the gut against harmful antigens and in providing a physiological barrier against the harsh luminal environment. ${ }^{13}$ However, the protective mechanisms involved are poorly defined. Among a number of the components of mucus, mucins are believed to have a major role. ${ }^{4}$ The high molecular weight mucins are responsible for the viscoelastic properties of the mucous barrier. They are widely expressed in epithelial tissues and are characterised by variable number tandem repeat peptide sequences rich in serine, threonine, and proline which carry large numbers of $\mathrm{O}$ linked oligosaccharide chains. To date, 13 mucins have been identified and their functions and distribution have been extensively studied. ${ }^{313}$ Secreted and membrane associated forms have been found based on their function as extracellular viscous secretions or viscoelastic polymer gels, or location as membrane anchored molecules in the glycocalyx. Recently, we identified another mucin, Fc $\gamma \mathrm{BP}$, associated with goblet cells in the human small intestine and colon. ${ }^{140}$ Fc $\gamma \mathrm{BP}$ is completely distinct from known IgG FC receptors as well as known mucin glycoproteins, but it has a CGLCGN motif in its amino acid sequence that is conserved in MUC2, MUC5AC, MUC5B, and prepro-von Willebrand factor

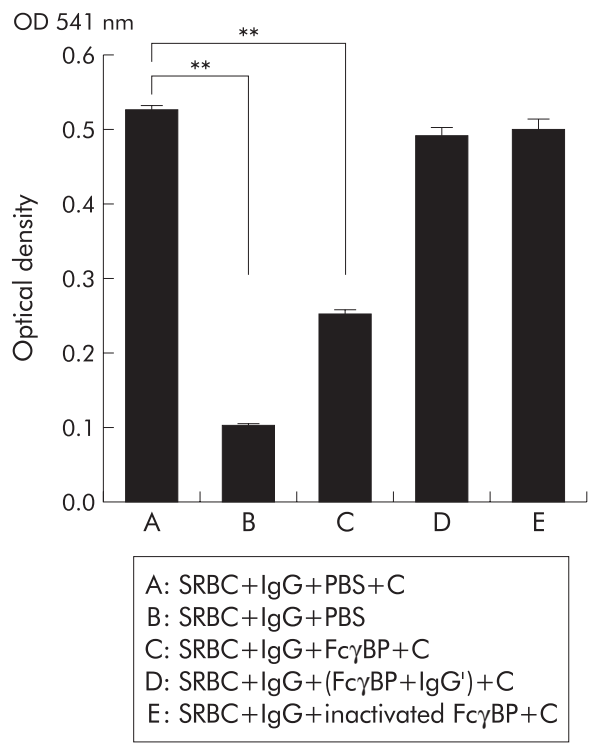

Figure 9 Inhibition of complement mediated haemolysis by lgG Fc binding protein (FcyBP). A: Optical density (OD) of the supernatant in the experiment of complement mediated haemolysis was 0.53 (0.02). B: OD of the supernatant in the experiment without complement was $0.10(0.002)$. C: $O D$ of the supernatant in the experiment with added FcyBP was 0.25 (0.01). D: OD of the supernatant in the experiment treated with an excess of non-immune rabbit lgG (to saturate $\mathrm{Fc \gamma BP}$ ) and $\mathrm{Fc}_{\mathrm{C}} \mathrm{BP}$ was 0.50 (0.02). E: OD of the experiment reacted with heat inactivated FcyBP was $0.51(0.03)$ From these results, it is evident that $\mathrm{Fc \gamma BP}$ specifically inhibits complement mediated haemolysis of erythrocytes. SRBC, sheep red blood cells; $\lg G$, anti-SRBC rabbit $\lg G$; $\lg G$, excess of non-immune rabbit lgG; PBS, phosphate buffered saline; C, complement.

but not in MUC6. ${ }^{3}{ }^{17}$ In addition, binding sites for IgG associated with MUC2 and TFF peptides have not yet been clarified. Because of the implications that Fc $\gamma \mathrm{BP}$ might be a physiologically important mucin, we felt it worthwhile to define the tissue distribution of $\mathrm{Fc} \gamma \mathrm{BP}$ and to begin characterising the activities of Fc $\gamma B$ P. Our major findings were that Fc $\gamma B$ P was widely distributed in mucus cells and secreted mucins. Furthermore, it retained IgG binding activity in several fluids and could inhibit a complement mediated reaction of IgG.

For use in the present study, we chose two mAbs (K9 and K17) to human Fc $\gamma B P$ from among 14 mAbs we had previously made. ${ }^{16}$ We had shown previously that both mAbs K9 and K17 strongly stained goblet cells in the human colon and inhibited in vitro binding of HRP-IgG to cells in tissue sections. ${ }^{16}$ The mAb K9 showed the highest titres in three types of ELISA to detect Fc $\gamma B$, and stained goblet cells under various conditions of fixation, including $10 \%$ formalin, $0.5 \%$ glutaraldehyde, and 100\% ethanol. Only mAb K17, among the mAbs which block binding of HRP-IgG in tissue sections, ${ }^{16}$ detected the 70-80 kDa band in immunoblot studies under reduced conditions. The material reactive with mAb K9 did not cross react with the propolypeptide of von Willebrand factor by amino acid sequence homology ${ }^{17}$ but the material reactive with mAb K17 did cross react with it. ${ }^{21}$ These antibodies did not react with MUC2, Muc5AC, MUC5B, or MUC6.

In the present study, we examined the existence and activity of Fc $\gamma \mathrm{BP}$ in various external body fluids and immunohistochemically identified the binding protein in various tissues. Initially, we found that gut lavage fluid did not have IgG binding activity when applied to a column of Sepharose linked to IgG. To determine whether various body fluids contain active Fc $\gamma B$ BP or only fragments of Fc $\gamma B$ P, a mixture of the fluids and mAbs were incubated with immunobeads (goat IgG antimouse $\operatorname{IgG}$ ), and the captured molecules were detected by mAb K17. Normal mouse IgG was unable to bind human 
FcyBP through its Fc portion, unlike human or goat IgG, as found in previous experiments. ${ }^{14}{ }^{16}$ As the Fc portion of mouse IgG had at least 128 times less affinity to human Fc $\gamma B P$ than the Fab portion of the $\mathrm{mAb}$ against $\mathrm{F}_{\mathrm{C}} \gamma \mathrm{BP}^{16}{ }^{16}$ non-specific binding of mouse IgG to Fc $\gamma \mathrm{BP}$ was negligible and fragments of $\mathrm{Fc} \gamma \mathrm{BP}$ were detected by the mAb as efficiently as was active Fc $\gamma$ BP.

In the stomach, we found that although some surface mucous cells were slightly stained with K9 and K17, HRP-IgG did not bind to cells. We suspect that this failure of immunoreactive FcyBP to bind HRP-IgG reflects degradation or damage of the FcyBP binding site(s) by the acidic gastric environment. Fc $\gamma \mathrm{BP}$ was detected by the immunodotblot assay in gastric juice with a pH of 6 or more. Thus Fc $\gamma B P$ may be secreted by the normal stomach but we cannot be certain that Fc $\gamma \mathrm{BP}$ detected by the immunodotblot in presumably normal gastric juice had not been secreted by gastric intestinal metaplasia cells or was present in duodenal juice or bile that had refluxed into the stomach. Recently, 13 types of mucins have been reported and the normal stomach is characterised by expression of MUC1, MUC5AC, and MUC6. ${ }^{18}{ }^{19} 22$ Expression of mucin is different in different types of intestinal metaplasia, and Helicobacter pylori infection alters expressions of mucin. ${ }^{22}{ }^{23}$ Although we did not examine our patients for $H$ pylori, all five specimens from patients with gastric cancer had intestinal metaplasia and the distribution of FcyBP was almost negative in apparently normal gastric mucosa adjacent to intestinal metaplasia. In addition, surface mucus cells in the apparently normal stomach adjacent to intestinal metaplasia did not react with HRP-IgG but these cells were weakly stained with mAbs in three of five samples. Thus variations in the environment of the stomachs of our patients may explain the variability in the results of Fc $\gamma \mathrm{BP}$ in the gastric samples. Further investigations on the expression and distribution of FcyBP in relation to $H$ pylori infection and the types of intestinal metaplasia are warranted to clarify the role of Fc $\gamma \mathrm{BP}$ in the stomach.

Mucus cells of the conjunctiva did not bind to HRP-IgG, and FcyBP was not detected in tears, even though some mucins (MUC1, MUC4, and MUC5AC) ${ }^{24}$ and mucin associated trefoil peptides (pS2 and intestinal trefoil factor ${ }^{25}$ are present on the ocular surface, while the ocular surface does not express less mucins than the gastric epithelium (MUCl, MUC6, and MUC5AC) or the colonic surface (MUC1, MUC2, MUC3, and MUC4). ${ }^{324}$ Although the reason why Fc $\gamma B P$ is not expressed on the ocular surface is unknown, it may be that the conjunctiva or cornea comes from an embryologically different origin (ectoderm) from that of the epithelium of the digestive or respiratory tracts (endoderm).

Fc $\gamma B$ P was originally identified in intestinal goblet cells and demonstrated to be secreted with mucus into the intestinal lumen. ${ }^{15}$ In fact, we found IgG activity and molecules detected by mAbs abundant in the mucus obtained from the surface of the large intestine, whereas IgG binding activity was not detected in the lavage fluid collected at endoscopy of the large intestine. However, immunoprecipitation detected the degraded smaller molecules in addition to the 70-80 kDa molecule. These findings may indicate that FcyBP released into the intestinal lumen is degraded by digestive enzymes resulting in inhibition of $\operatorname{IgG}$ binding activity. In contrast, Fc $\gamma \mathrm{BP}$ is not degraded in nasal discharges, sputum, or bile. Fc $\gamma$ BP strongly binds aggregated IgG and IgG complexes. ${ }^{17}$ Thus we have speculated that Fc $\gamma \mathrm{BP}$ helps to protect the gut mucosal surface by binding IgG molecules in complexes with antigens, including infectious microorganisms. This activity may facilitate clearance of the complexes from mucosal surfaces. If this were the case, degradation of Fc $\gamma \mathrm{BP}$ in gut secretions may seem to be counterproductive. However, it is possible that FcyBP is not degraded once complexed with IgG.

Another important finding of this work was that FcyBP inhibited complement mediated haemolysis of SRBC. Comple- ment mediated reactions involving IgG molecules on mucosal surfaces or in external secretions may be injurious. Thus a mechanism for inhibiting such reactions might be beneficial. Normally, not much IgG is present in external secretions. ${ }^{14}$ However, as in inflammatory diseases, Fc $\gamma B$ BP may prevent injurious complement mediated reactions from occurring by binding any available IgG. Interestingly in this regard, we found increased amount of Fc $\gamma B$ P antigen in the sera of patients with inflammatory bowel diseases and autoimmune diseases..$^{21}$ Taken together, we suggest that $\mathrm{F} c \gamma \mathrm{BP}$ plays a role in the protection of tissue damage by immune complexes in the intestinal wall.

In summary, our findings that Fc $\gamma \mathrm{BP}$ is present widely throughout mucous membranes and in external body fluids, that FcyBP retains IgG binding activity in many fluids, and that Fc $\gamma B P$ can inhibit a complement mediated reaction, suggest that this novel mucin is important in the immunological defence of mucosal surfaces. Further study of the biological importance of Fc $\gamma \mathrm{BP}$ is warranted.

\section{ACKNOWLEDGEMENTS}

This study was supported in part by grants in aid from the Japanese Ministry of Education, Culture and Science; the Japanese Ministry of Health and Welfare; and Keio University, Tokyo, Japan.

\section{Authors' affiliations}

K Kobayashi, H Ogata, N Inove, Y Hamada, H Ishii, M Watanabe,

T Hibi, Department of Internal Medicine, School of Medicine, Keio University, Tokyo, Japan

M Morikawa, S lijima, N Harada, T Yoshida, Chugai Pharmaceutical Co, Ltd, Tokyo, Japan

W R Brown, Gastroenterology Division, University of Colorado Health Sciences Center and Department of Veterans Affairs Medical Center, Denver, CO, USA

\section{REFERENCES}

1 Allen A, Hutton DA, Leonard AJ, et al. The role of mucus in the protection of the gastrointestinal mucosa. Scand J Gastroenterol 1986;21:71-7

2 Owen DA, Kelly JK. Inflammatory diseases of the gastrointestinal tract. Mod Pathol 1995:8:97-108.

3 Corfield AP, Myerscough N, Longman R, et al. Mucins and mucosal protection in the gastrointestinal tract: new prospects for mucins in the pathology of gastrointestinal disease. Gut 2000;47:589-94.

4 Filipe MI. Mucins in the human gastrointestinal epithelium: a review. Invest Cell Pathol 1979:2:195-216.

5 Specian RD, Oliver MG. Functional biology of intestinal goblet cells. Am J Physiol 1991;260:C183-93.

6 Verma M, Davidson EA. Mucin genes: structure, expression and regulation. Glycoconj J 1994;11:172-9.

7 Pandey $\mathbf{P}$, Kharbanda S, Kufe D. Association of the DF3/MUC1 breast cancer antigen with Grb2 and the Sos/Ras exchange protein. Cancer Res 1995;55:4000-3

8 Yamamoto $M$, Bharti A, Li Y, et al. Interaction of the DF3/MUC1 breas carcinoma-associated antigen and beta-catenin in cell adhesion. J Biol Chem 1997;272:12492-4.

9 Li YQ, Bharti A, Chen DS, et al. Interaction of glycogen synthase kinase $3 \beta$ with the DF3/MUC 1 carcinoma-associated antigen and $\beta$-catenin Mol Cell Biol 1998;18:7216-24

10 Slomiany BL, Murty VLN, Piotrowski J, et al. Salivary mucins in oral mucosal defense. Gen Pharmacol 1996;27:761-71.

11 Lillehoi EP, Hyun SW, Kim BT, et al. Mucl mucins on the cell surface are adhesion sites for Pseudomonas aeruginosa. Am J Physiol 2001;280:L181-7

12 Carraway KL, Rossi EA, Komatsu M, et al. An intramembrane modulator of the ErbB2 receptor tyrosine kinase that potentiates neuregulin signaling. J Biol Chem 1999;274:5263-6.

13 Williams SJ, Wreschner DH, Tran M, et al. MUC13, a novel human cell surface mucin expressed by epithelial and hemopoietic cells. J Biol Chem 2001:276:18327-36

14 Kobayashi K, Blaser M, Brown WR. Identification of a unique lgG Fc binding site in human intestinal epithelium. J Immunol 1989;143:256974.

15 Hamada Y, Kobayashi K, Podolsky DK, et al. Increased cytoplasmic accumulation of goblet cell glycoproteins in ulcerative colitis. Inflamm Bowel Dis 1996;2:97-104. 
16 Kobayashi K, Hamada $Y$, Blaser M, et al. The molecular configuration and ultrastructural location of an $\lg G$ Fc binding side in human colonic epithelium. J Immunol 1991;146:68-74.

17 Harada N, lijima S, Kobayashi K, et al. Human lgG Fc binding protein $(\mathrm{Fc} \gamma \mathrm{BP})$ in colonic epithelial cells exhibits mucin-like structure. J Biol Chem 1997:272:15232-41.

18 Gendler SJ, Spicer AP. Epithelial mucin genes. Annu Rev Physiol 1995;57:607-34.

19 Van Klinken BJ-W, Dekker J, et al. Mucin gene structure and expression: protection vs. adhesion. Am J Physiol 1995:269:G613-27.

20 Kobayashi K, Brown WR. Study of colonic lgG Fc binding site in cultured epithelial cells. Dig Dis Sci 1994;39:526-33.
21 Kobayashi K, Yagasaki M, Harada N, et al. Detection of Fc $\gamma$ binding protein antigen in human sera and its relation with autoimmune diseases. Immunol Lett 2001;79:229-35.

22 Ho SB, Shekels LL, Toribara NW, et al. Mucin gene expression in normal, preneoplastic, and neoplastic human gastric epithelium. Cancer Res 1995;55:2681-90

23 Ota H, Nakayama J, Momose M, et al. Helicobacter pylori infection produces reversible glycosylation changes to gastric mucins. Virchows Arch 1998:433:419-26.

24 Uebermuth C. Mucins of the eye. Ophthalmologie 1999:96:563-9.

25 Langer G, Jagla W, Behrens-Baumann W, et al. Secretory peptides TFF and TFF3 synthesized in human conjunctival goblet cells. Invest Ophthalmol Vis Sci 1999;40:2220-4.

\section{Clinical Evidence-Call for contributors}

Clinical Evidence is a regularly updated evidence based journal available world wide both as a paper version and on the internet. Clinical Evidence urgently needs to recruit a number of new contributors. Contributors are health care professionals or epidemiologists with experience in evidence based medicine and the ability to write in a concise and structured way.

We are presently interested in finding contributors with an interest in the following clinical areas:

Acute bronchitis

Hepatitis B

Acute sinusitis Hepatitis C

Cataract HIV

Genital warts

Being a contributor involves:

- Appraising the results of literature searches (performed by our Information Specialists) to identify high quality evidence for inclusion in the journal.

- Writing to a highly structured template (about 1500-3000 words), using evidence from selected studies, within 6-8 weeks of receiving the literature search results.

- Working with Clinical Evidence Editors to ensure that the text meets rigorous epidemiological and style standards.

- Updating the text every eight months to incorporate new evidence.

- Expanding the topic to include new questions once every 12-18 months.

If you would like to become a contributor for Clinical Evidence or require more information about what this involves please send your contact details and a copy of your CV, clearly stating the clinical area you are interested in, to Polly Brown (pbrown@bmigroup.com). 